\title{
Endometriosis fertility index score maybe more accurate for predicting the outcomes of in vitro fertilisation than r-AFS classification in women with endometriosis
}

Wenjun Wang ${ }^{1 *}$, Ruiqi Li ${ }^{1}$, Tingfeng Fang ${ }^{1}$, Lili Huang ${ }^{2}$, Nengyong Ouyang ${ }^{1}$, Liangan Wang ${ }^{3}$, Qingxue Zhang ${ }^{1}$ and Dongzi Yang ${ }^{1}$

\begin{abstract}
Background: Endometriosis is a common disease. The most widely used staging system of endometriosis is the revised American Fertility Society classification (r-AFS classification) which has limited predictive ability for pregnancy after surgery. The endometriosis fertility index (EFI) is used to predict fecundity after endometriosis surgery. This diagnostic accuracy study was designed to compare the predictive value of the EFI with that of the r-AFS classification for IVF outcomes in patients with endometriosis.

Methods: 199 women with endometriosis receiving IVF treatment after surgery were analysis. The EFI score and r-AFS classification in their ability to predict these IVF outcomes were compared in the same population. ROC curves were used to analyse the predictive values of the EFI and r-AFS indices for clinical pregnancy, and their accuracies were evaluated by sensitivity, specificity, and the Youden's index.

Results: The Area Under the Curve (AUC) of the EFI score (AUC $=0.641$, Standard Error(SE) $=0.039, P=0.001$, $95 \% \mathrm{Cl}=0.564-0.717$, cut-off score $=6$ ) was significantly larger than that of the r-AFS classification (AUC $=0.445$, $\mathrm{SE}=0.041, \mathrm{P}=0.184$, and $95 \% \mathrm{Cl}=0.364-0.526$ ). The antral follicle count, oestradiol level on day of $\mathrm{hCG}$, number of oocytes retrieved, number of oocytes fertilised, and number of cleaved embryos in the greater than or equal to $6 \mathrm{EFI}$ score group was greater than that of the lower than or equal to $5 \mathrm{EFI}$ score group, and the dose of gonadotropin of the greater than or equal to $6 \mathrm{EFI}$ score group were less than that in the lower than or equal to $5 \mathrm{EFI}$ score group. Implantation rate, clinical pregnancy rate, and cumulative pregnancy rate in the greater than or equal to $6 \mathrm{EFI}$ score group were higher than in the lower than or equal to $5 \mathrm{EFI}$ score group.

Conclusions: It suggests that the EFI has more predictive power for IVF outcomes in endometriosis patients than the r-AFS classification. The clinical pregnancy rate was higher in patients with EFI greater than or equal to 6 score than with EFI lower than or equal to 5 score.
\end{abstract}

Keywords: Endometriosis fertility index (EFI), Revised American Fertility Society (r-AFS) classification, Endometriosis, In vitro fertilization (IVF), Clinical pregnancy rate

\footnotetext{
* Correspondence: wenjungzcn@163.com

'Department of Obstetrics \& Gynecology, Reproductive medicine centre, Sun Yat-Sen Memorial Hospital, Sun Yat-Sen University, No.107 Yanjiang Xi Road, Guangzhou 510120, P. R. China

Full list of author information is available at the end of the article
} 


\section{Background}

Endometriosis is a common disease that occurs in 6 to $10 \%$ of reproductive-age women [1,2]. Approximately 25 to $50 \%$ of infertile women have endometriosis, and 30 to $50 \%$ of women with endometriosis are infertile [3]. Today, the most widely used staging system of endometriosis is the revised American Fertility Society classification ( $r$-AFS classification) $[4,5]$. The $r$-AFS classification is used to predict the recurrence potential of endometriosis after surgery. However, it has limited predictive ability for pregnancy after surgery. Adamson and Pasta suggested that the r-AFS classification depends mainly on morphological descriptions [6], whereas Vercellini et al. observed no association between the endometriosis stage or lesion type and lesion site and the cumulative probability of pregnancy [7].

The endometriosis fertility index (EFI), proposed by Adamson and Pasta in 2010, is used to predict fecundity after endometriosis surgery [8]. In addition to providing a detailed score to the appendix (fallopian tubes, fimbriae of fallopian tubes, ovaries) by calculating the leastfunction scores, the EFI also combines conceptionrelated factors such as age, duration of infertility, and gravidity history. The EFI contains all of the components of the r-AFS stage score, but the r-AFS score includes only $20 \%$ of the EFI score. However, the EFI does not consider whether the patient has received in vitro fertilisation (IVF) treatment after endometriosis surgery [9]. Until now, there is no report on the evaluation of IVF outcomes and embryo quality by the EFI score after endometriosis surgery, and no report was on the comparison of the EFI score with the r-AFS classification in the prediction of IVF outcome. This study aimed to compare the predictive value of EFI score with that of $r$ AFS classification in the same population of women with endometriosis who had received IVF treatment. We performed a retrospective analysis of the case histories, operative records, and the available information on embryo quality, implantation rate, and pregnancy rate of infertile women who had received IVF treatment after endometriosis surgery.

\section{Methods}

\section{Study population and design}

The study was conducted after receiving Institutional Review Board approval. Patients were provided with counseling, and signed consents were obtained.

A total of 199 women of reproductive age who had endometriosis and received their first cycle of IVF treatment between January 2008 and July 2012 were enrolled in this study. All cases had a histological diagnosis of endometriosis and received laparoscopic surgery before IVF treatment. Patients who failed to get pregnant in at least the first 6 months after laparoscopic surgery, although they had intercourse at least twice a week, were eligible to receive IVF-embryo transfer (ET) treatment. The results of semen examination of patients' husbands were within the World Health Organization (WHO) reference range [10]. No couple used any contraception. In addition, the patients did not receive any ovulation stimulation treatment within 6 months before the IVF treatment. The exclusion criteria (determined by laparoscopy, ultrasound examination, and hormonal tests) were uterine myoma, adenomyoma, congenital structural abnormalities of the reproductive tract, pelvic tuberculosis, ovarian tumour, polycystic ovary syndrome, hyperprolactinaemia, adrenal disease, thyroid disease or other endocrine disease, and male infertility.

\section{r-AFS classification method}

According to the standards of r-AFS classification (1985 and 1996) $[4,5]$ the lesion score and total score were calculated based on a retrospective analysis of the surgery report in the hospital medical records. The endometriosis stage of each patient was classified as well. We obtained institutional review board approval for retrieving the laparoscopic surgery records and the IVF data.

\section{EFI score}

The EFI score was calculated according to the EFI developed by Adamson and Pasta [8]. It includes the following clinical and surgical factors (Figure 1): age, duration of infertility (years), pregnancy history, least-function (LF) score (including fallopian tubes, tubal fimbriae, and ovaries; LF score $=$ the least score of the left side + the least score of the right side; if any ovary was absent, the LF score was obtained by doubling the LF score of the contralateral side), $r$ AFS score of the lesion, and total r-AFS score. Figure 1 shows Rating scale of the least-function score and the Endometriosis Fertility Index

\section{Ovarian stimulation, insemination, and IVF treatment}

The prolonged gonadotropin-releasing hormone agonist protocol was performed as follows. The patients underwent pituitary down-regulation through an intramuscular injection of $3.75 \mathrm{mg}$ of triptorelin acetate (Diphereline; Ipsen Pharma Biotech. France) [11,12]. Twenty-eight to 35 days later, the patients were given a second injection of $1.25 \mathrm{mg}$ (one third of Diphereline ${ }^{\circledR}$ ) of triptorelin acetate. Twentyone to 28 days later, the patients were given a third injection of $0.93 \mathrm{mg}$ (quarter of one Diphereline ${ }^{\circledR}$ ) of triptorelin acetate. Then, 1 week later, recombinant follicle-stimulating hormone $(\mathrm{rFSH})$ was used to stimulate follicle development. The initial dose of rFSH (Gonal-F,75 IU/per amp, Merck Serono, Geneva, Switzerland) was 150-300 IU/day, depending on the antral follicle count (AFC), patient age, and basal serum level of FSH. Follicle development was monitored under transvaginal ultrasound guidance. 


\section{ENDOMETRIOSIS FERTILITY INDEX (EFI) SURGERY FORM}

LEAST FUNCTION (LF) SCORE AT CONCLUSION OF SURGERY

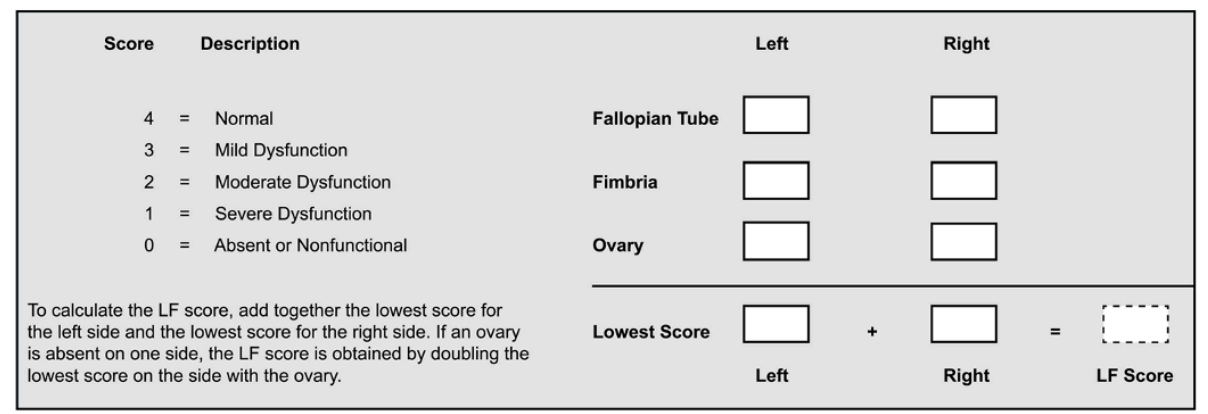

ENDOMETRIOSIS FERTILITY INDEX (EFI)

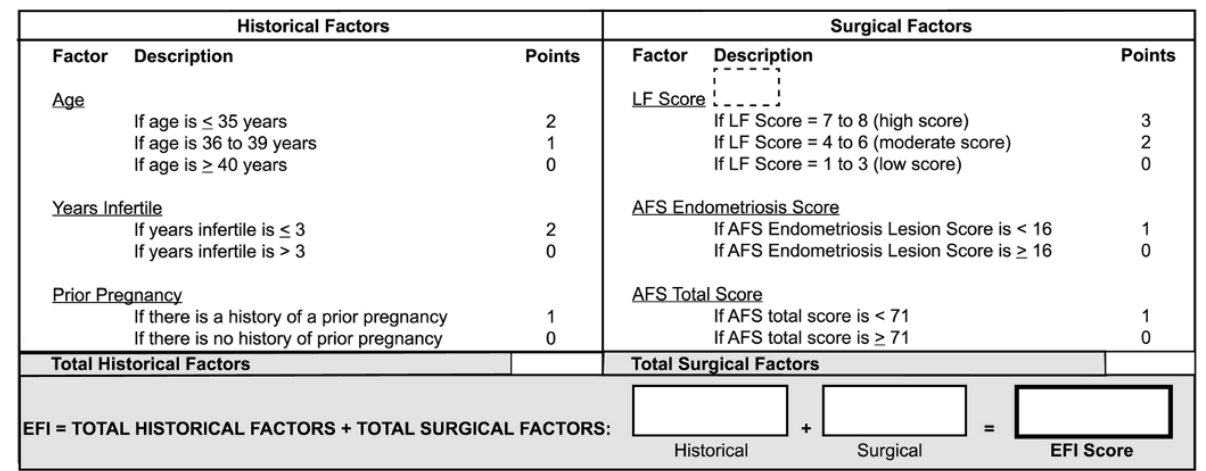

ESTIMATED PERCENT PREGNANT BY EFI SCORE

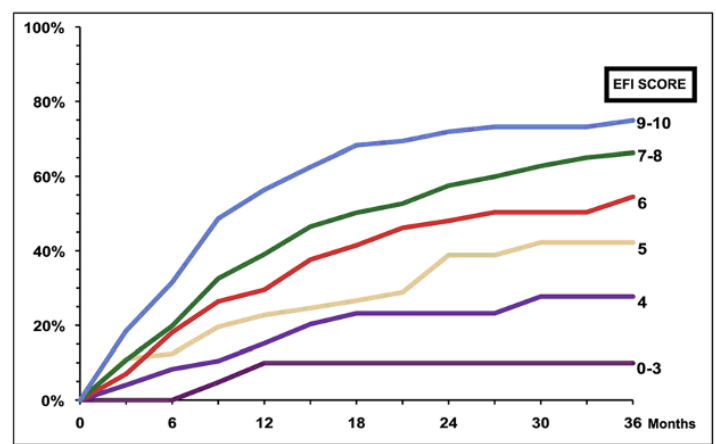

Figure 1 Rating scale of the least-function score and the Endometriosis Fertility Index. Note: The Table is from Adamson GD, Pasta DJ. Endometriosis fertility index: the new, validated endometriosis staging system. Fertil Steril. 2010. 94(5): 1609-1615 [8]. Copyright 2010, with permission from Elsevier.

Recombinant human chorionic gonadotropin (hCG) (Ovidrel, Serono) (250 mg) was administered subcutaneously when two or more follicles $\geq 18 \mathrm{~mm}$ in mean diameter were present. Oocytes were retrieved by puncture of the vaginal sac guided by transvaginal ultrasound 35 to 36 hours after hCG administration [13]. Insemination was performed with 150,000 motile sperms cells/mL, always between 14:00 and 15:00 on the day of oocyte retrieval. Fertilisation was assessed 16 to 18 hours after insemination by searching the oocytes for evidence of pronucleus formation and a second polar body. Each embryo was incubated in a separate droplet of G1 medium (VitroLife ${ }^{\circledR}$, Sweden ) covered with paraffin oil (VitroLife ${ }^{\circledR}$, Sweden ).

Embryo transfer was performed on the third day after retrieval, and the luteal phase was supplemented with injection of $40 \mathrm{mg}$ of progesterone/day. Biochemical pregnancy was confirmed by measuring serum $\beta$-hCG on the 14th day after transfer. Clinical pregnancy was confirmed by the presence of a gestational sac and foetal heart beat on the 30th to 35 th day after transfer. 


\section{Embryo evaluation}

Fertilisation and pronuclear morphology were assessed 16-18 hours after insemination. Zygotes were checked for the number, size, and position of the pronuclei (PN) as well as the number and location of the nucleolar precursor bodies within the $\mathrm{PN}$, as described previously by Scott et al [14].

The assessment on days 2 and 3 was performed as follows. Morphologic assessments were always performed at the same time (between 8:00 am and 9:30 am). The embryos were identified as grade I (good quality) if they had either 2-4 blastomeres of equal size and less than $20 \%$ fragmentation on day 2 or 7-8 blastomeres of equal size and less than $20 \%$ fragmentation on day 3 . The embryo transfer was performed on the third day after oocyte collection [15]. Three embryos were transferred in the patients who were 35 years or older, and one or two embryos were transferred in the patients who were younger than 35 years. Clinical pregnancy was determined by the presence of a gestational sac and heart beat by a transvaginal ultrasound performed on the $30^{\text {th }}$ to $35^{\text {th }}$ days after the embryo transfer. The culture medium was G5 series(VitroLife ${ }^{\circledR}$, Sweden).

\section{Hormone assays}

Blood samples were obtained from each patient with regular menstruation prior to the initiation of a stimulation cycle, at 9:00 am-10:00 am during days 2 to 4 of the menstrual cycle. A Beckman Coulter UniCel DxI 800 and the associated reagents (Beckman Coulter, Los Angeles, USA) were used to determine the serum sex hormone levels (basal FSH, basal luteinising hormone, basal oestradiol) by chemiluminescence.

\section{Data analysis and statistical methods}

Estimation of sample size: To compare the EFI score and $\mathrm{r}$-AFS classification on the fecundity and IVF outcomes, we used a diagnostic test to estimate the sample size. Because the two indices would be used on the same population, we used the EFI score to calculate the sample size. The pregnancy state after IVF was the gold standard, and positive meant pregnancy and negative meant non-pregnancy.

A total of 161 cases were collected from January 2008 to December 2011, who were diagnosed with endometriosis and received IVF treatment after laparoscopy. After the area under the receiver operating characteristic curve (AUC ROC) for EFI score was calculated, the cutoff score was 6 . Therefore, the 161 cases were divided into two groups, a $\leq 5$ score group and a $\geq 6$ score group. We next calculated the necessary sample size as the following formula Formula for sample size estimation).

To avoid excessive variability and minimise bias, we paid close attention to the following before calculating the sample size. Sample drawing: the non-pregnant women with endometriosis were enrolled 6 months after laparoscopic surgery if they had requested IVF treatment. The gold standard of the diagnostic test was 'pregnancy'. We chose an accuracy index $\alpha=0.05$, power $=$ $90 \%$, and a bilateral variability test. All the surgeries were performed by three highly experienced gynaecologic surgeons who worked in the same hospital and the assessments of the endometriosis classification staged were also performed by these three surgeons. They had concordant opinions and judgments of endometriosis traits. The scores from their operation records were comparable.

Formula for sample size estimation [16]:

$$
\begin{gathered}
n=\left\{\frac{Z_{1-a / 2} \sqrt{2 \bar{p}}+Z_{1-\beta} \sqrt{2\left(p_{1}-p\right)\left(p_{1}-p\right) / \bar{p}}}{p_{1}-p_{2}}\right\}^{2} \\
p_{1}=\frac{a+b}{N} \\
p_{2}=\frac{a+b}{N} \\
p=\frac{a}{N} \\
\bar{p}=\frac{p_{1}+p_{2}-2 p}{2}
\end{gathered}
$$

Data collection: We performed a retrospective analysis of the operation records and the case records from the medical record archive file, medication doses during IVF treatment, number of oocytes retrieved, fertilisation rate, embryo development, and pregnancy rate.

Data calculation: The sample size $(\mathrm{n}=196)$ was determined by the data in Table 1 and above formula for sample size estimation [16,17]. Table 1 shows sample size estimation using the diagnostic test.

After the sample size was determined, the cases were continuously collected until 31 July 2012. A total of 199 cases were enrolled. ROC curves were used to analyse the predictive values of the EFI and r-AFS indices for clinical pregnancy, and their accuracies were evaluated by sensitivity, specificity, and Youden's index. The ROC analysis was used to calculate the cut-off point of each index for successful prediction. The ROC of the EFI score and the ROC of $\mathrm{r}$-AFS classification were first drawn in SPSS v20.0, and the cut-off point of each diagnosis was calculated. The AUC of the EFI score and the AUC of r-AFS were compared. The cases were divided

Table 1 Sample size estimation using the diagnostic test

\begin{tabular}{lccc}
\hline EFI & \multicolumn{2}{c}{ Pregnancy } & Total \\
\cline { 2 - 3 } score & Positive & Negative & \\
\hline$\geq 6$ score & $a(44)$ & $b(47)$ & $a+b(91)$ \\
$\leq 5$ score & $c(23)$ & $d(47)$ & $c+d(70)$ \\
Total & $a+c(67)$ & $b+d(94)$ & $N(161)$ \\
\hline
\end{tabular}


into two groups according to the EFI score $(\leq 5$ and $\geq 6)$. The same populations were divided into two groups according to the r-AFS classification (stage I-II and stage III-IV). The goodness of fit of each diagnostic system was assessed by the AUC ROC, in which a higher AUC value indicated a better fit to predict implantation. Significance was defined as a two-tailed $P$ value less than 0.05 .

The statistical analyses were performed using SPSS v20.0 software. The normally distributed data of quantitative variation and continuous variables are reported as the mean \pm standard deviation and were compared using the $t$-test. The non-normally distributed data are reported as the median (inter-quartile range) and were compared using the Mann-Whitney test. Comparison between the two EFI groups and between the two r-AFS groups was performed with the chi-squared test for categorical variables or the $t$-test and Mann-Whitney test for continuous and ordinal variables. All tests were two tailed, and $\mathrm{P}<0.05$ was considered statistically significant. Whenever appropriate, writing and analysis followed the STARD [18].

\section{Results}

Baseline and cycle characteristics of the 199 patients who were enrolled in the study (Table 2):

The average age was $32.0 \pm 4.2$ years. The regular menstruation period was 27 to 33 days and lasted 5 to 7 days. The average body mass index (BMI) was $20.2 \mathrm{~kg} / \mathrm{m}^{2}$. Fifty-two patients had received ovarian stimulation therapy once before laparoscopic surgery, and 135 had primary infertility and 64 secondary infertility. Five patients with secondary infertility had experienced an ectopic pregnancy, and three of these five cases had a history of elective abortion before ectopic pregnancy. Fifteen patients had once spontaneous miscarriage, and 44 had received an elective abortion. All 199 women had attempted to conceive by natural methods and were unsuccessful after 6 months and so requested IVF treatment. The outcome of IVF was that 85 women achieved pregnancy in the first, fresh cycle, and 107 women achieved pregnancy after a fresh cycle and a freeze-thaw cycle. The cumulative pregnancy rate was $53.8 \%$.

\section{ROCs for pregnancy diagnosis of the EFI and r-AFS indices}

The 199 fresh IVF-ET cycles were analysed in this retrospective study. In fresh IVF-ET cycles, 85 cycles resulted in pregnancy and 114 cycles resulted in non-pregnancy. The EFI showed an AUC $=0.641$, Standard Error $(\mathrm{SE})=$ $0.039, \mathrm{P}=0.001,95 \%$ Confidence Interval $(\mathrm{CI})=0.564-$ 0.717 , cut-off score $=6$ score, sensitivity $=71.8 \%$, specificity $=52.6 \%$, and Youden index $=0.244$ (Youden index $=$ sensitivity + specificity-1). The r-AFS classification showed an $\quad \mathrm{AUC}=0.445, \quad \mathrm{SE}=0.041, \quad \mathrm{P}=0.184, \quad$ and $\quad 95 \%$ $\mathrm{CI}=0.364-0.526$.

As shown in "ROC of pregnancy in fresh IVF cycles for the EFI score and r-AFS classification" subsection, the AUC of the EFI score was larger than that of the r-AFS classification. This suggests that the EFI score had a greater ability to predict IVF pregnancy outcome than r-AFS.

Using the cut-off EFI score of 6, we compared each IVF item between the $\leq 5$ EFI score group and the $\geq$ EFI 6 score group. We performed a similar comparison for each IVF item between the r-AFS stage I-II group and the stage III-IV group. Figure 2 shows ROC of pregnancy in fresh IVF cycles for the EFI score and r-AFS classification.

Comparison between the two EFI score groups and between the two r-AFS stage groups (Tables 2 and 3).

First, baseline and IVF cycle characteristics of the patients in the two EFI groups were compared by the $t$-test and Mann-Whitney test. Then, the medication doses during IVF treatment, embryo development, and IVF outcomes were analysed. Patient age and duration of infertility are included in the EFI score, and the patient age in the $\leq 5$ score group was greater than that in the $\geq 6$ score group. The duration of infertility in the $\leq 5$ score group was longer than that in the $\geq 6$ score group. Among the factors we used to evaluate the ovarian reserve in the EFI score, the AFC in the $\geq 6$ score group was greater than that of the $\leq 5$ score group, and the starting dose and total dose of $\mathrm{Gn}$ of the $\geq 6$ score group were less than those of the $\leq 5$ score group. The effectiveness of ovarian stimulation in the $\geq 6$ score group was better than that in the $\leq 5$ score group. The oestradiol level of the day of hCG and the number of oocytes retrieved were greater in the $\geq 6$ score group. The number of two-pronucleus (2PN) fertilisations and the number of cleavages from $2 \mathrm{PN}$ were greater in the $\geq 6$ score group. Implantation rate, clinical pregnancy rate, and $\mathrm{cu}-$ mulative pregnancy rate were also higher in the $\geq 6$ score group.

We next compared the various factors between the r-AFS stage I-II and stage III-IV groups. There was no significant difference between groups in the factors that affect ovarian reserve, including basal FSH and AFC. The number of oocytes retrieved and the number of fertilisations were greater in the stage I-II group than in the stage III-IV group. However, the availability of embryos was lower in the stage I-II group. There were no significant differences in implantation rate, clinical pregnancy rate, or accumulation rate of pregnancy between the two groups.

Among the 199 cases, 386 embryos were transferred. There were 53 cycles with singletons, 29 cycles with twin pregnancies, and 3 cycles with triplet pregnancies. 


\section{Table 2 Baseline and cycle characteristics of the patients}

\begin{tabular}{|c|c|c|c|c|c|c|c|c|c|}
\hline \multirow[t]{2}{*}{ Characteristic } & \multirow[t]{2}{*}{ Total } & \multicolumn{4}{|c|}{ EFI } & \multicolumn{4}{|c|}{ r-AFS } \\
\hline & & $\leq 5$ score & $\geq 6$ score & $t(z)$ & $P^{c}$ & I-II stage & III-IV stage & $t(z)$ & $P^{d}$ \\
\hline No. of cycles & 199 & 84 & 115 & & & 61 & 138 & & \\
\hline Average age $(y)^{a}$ & $32.0 \pm 4.2$ & $33.3 \pm 4.9$ & $31.1 \pm 3.3$ & 3.789 & 0.000 & $32.5 \pm 3.7$ & $31.8 \pm 4.4$ & 1.085 & 0.279 \\
\hline Duration of infertility $(y)^{b}$ & $5.0(3.0-7.0)$ & $6.0(4.0-9.0)$ & $4.0(2.0-6.0)$ & -3.893 & 0.000 & $5.0(3.5-7.0)$ & $4.0(2.0-7.0)$ & -1.176 & 0.240 \\
\hline BMI $\left(\mathrm{kg} / \mathrm{m}^{2}\right)^{\mathrm{b}}$ & $20.2(18.8-21.7)$ & $20.2(18.2-22.0)$ & 20.3(18.9-21.7) & -0.374 & 0.708 & 20.6(19.1-21.8) & $20.0(18.5-21.6)$ & -1.013 & 0.311 \\
\hline bFSH(iu/L) $)^{b}$ & $8.1(6.7-9.9)$ & $8.6(6.9-11.7$ & $8.0(6.6-9.3)$ & -1.645 & 0.100 & $7.9(6.3-9.3)$ & $8.3(6.9-11.0)$ & -1.683 & 0.092 \\
\hline bLH(iu/L) $)^{b}$ & $3.8(2.7-5.5)$ & $3.8(2.7-5.3)$ & $3.8(2.9-5.6)$ & -0.009 & 0.993 & $3.7(2.7-5.5)$ & $4.0(2.9-5.5)$ & -0.706 & 0.480 \\
\hline$E 2(n g / L)^{b}$ & $42.4(27.0-61.9)$ & $47.4(29.7-57.9)$ & $42.0(27.3-64.7)$ & -0.957 & 0.339 & 42.0(22.9-64.3) & $45.9(27.1-60.5)$ & -0.864 & 0.388 \\
\hline Antral follicle count ${ }^{b}$ & $10(6.0-14.0)$ & $8.5(6.0-13.0)$ & $11.0(8.0-14.0)$ & -2.212 & 0.027 & $10.0(8.0-14.0)$ & $9.5(6.0-14.0)$ & -1.662 & 0.097 \\
\hline E2 level on $D_{H C G}(n g / L)^{b}$ & 1987.2(1122.6-3369.1) & $1661.3(925.2-3599.5)$ & $2110.3(1239.8-3334.0)$ & -1.255 & 0.209 & $2352.6(1331.7-4241.5)$ & $1796.9(1012.2-3251.3)$ & -2.065 & 0.039 \\
\hline Start dose of Gn(iu/day) $)^{b}$ & $225.0(150.0-300.0)$ & $225.0(150.0-300.0)$ & $225.0(150.0-225.0)$ & -2.401 & 0.016 & $225.0(150.0-225.0)$ & $225.0(150.0-300.0)$ & -1.853 & 0.064 \\
\hline Duration of stimulation $(\mathrm{d})^{\mathrm{b}}$ & $10(9.0 \sim 12.0)$ & 10(9.0-12.0) & 10(9.0-12.0) & -0.497 & 0.619 & $11(9-11)$ & 10(9-12) & -0.214 & 0.830 \\
\hline Total dose of Gn(iu) ${ }^{a}$ & $2286.6 \pm 926.4$ & $2439.3 \pm 1076.8$ & $2175.0 \pm 785.3$ & -1.909 & 0.058 & $2169.6 \pm 784.5$ & $2338.3 \pm 980.8$ & -1.186 & 0.237 \\
\hline
\end{tabular}

Note: The Shapiro-Wilk test was performed to determine the normality of distribution. $\mathrm{P}>0.05$ indicates the data followed a normal distribution.

${ }^{a}$ The normally distributed data are presented as mean $\pm S D$, including age and total dose of $\mathrm{Gn}$. The $t$-test was performed to analyse statistical significance.

${ }^{\mathrm{b}}$ The non-normally distributed data are presented as median (25th-75th percentile). The non-parametric Mann-Whitney test was performed to calculate the Z-score.

cComparison between the two EFI score groups (score $\leq 5$ vs score $\geq 6$ ).

dComparison between the two r-AFS stage groups (stage I-II vs stage III-IV). 


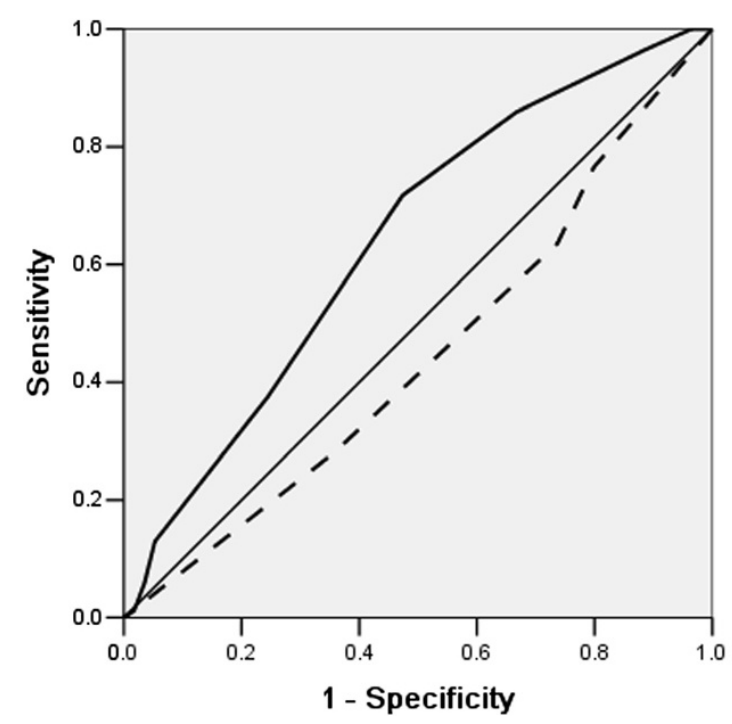

Source of the Curve

- AFS classification

- EFI

- Reference Line

1 - Specificity

Figure 2 ROC of pregnancy in fresh IVF cycles for the EFI score and r-AFS classification.

\section{Discussion}

The relationship between r-AFS classification and pregnancy rate in patients receiving IVF treatment after laparoscopic surgery

The r-AFS classification depends on the results of laparoscopic examination and laparotomy. The staging of endometriosis requires the detailed observation and recording of the site, number, size, and depth of the endometriosis lesions, as well as the degree of adhesions, to define the final score. The r-AFS score is mainly used to assess disease severity and develop a post-operative treatment plan. The diameter of a 'chocolate cyst' in the ovary plays a critical role in determining the $r$-AFS score. For patients with endometriosis who want to become pregnant, this staging method has limited ability to predict future fertility after surgery [8]. Opoien et al. [20]conducted a large retrospective study of r-AFS classification of endometriosis and the success rate of using IVF/intracytoplasmic sperm injection in treating female infertility due to fallopian tube disease. They found that there was no difference in pregnancy rate or childbirth rate between patients with different stages of endometriosis or fallopian tube diseases after patients with adenomyoma were excluded. Similarly, the present study excluded patients with adenomyoma and only evaluated the outcome of IVF in the treatment of infertility due to fallopian tube disease. Our results show that there was no statistical difference between the implantation rate and the pregnancy rate in patients with different stages of endometriosis, consistent with the above study. In addition, the AUC ROC of r-AFS was 0.445, which was not significant for diagnosis. Therefore, our data suggest that the r-AFS classification can predict neither future pregnancy in endometriosis patients nor the outcome of
IVF. We speculate that the EFI score maybe more sensitive in predicting pregnancy because it not only considers the size and number of lesions and the degree of local adhesion but also consider other reproductive factors, such as age, infertility duration, or fallopian tube and ovarian function.

The relationship between EFI score and pregnancy rate in patients receiving IVF treatment after laparoscopic surgery

In 2002, Fujushita et al. modified the AFS classification of endometriosis by adding the TOP score, (fallopian tubes, ovaries, peritoneum, and other factors) [21]. However, they did not consider the patient's age or other factors affecting pregnancy. Adamson and Pasta (2010) further revised and updated the AFS classification system. They prospectively collected detailed clinical and surgical data of 579 patients with endometriosis and analysed 275 variables associated with pregnancy, thereby establishing the EFI. In addition, they confirmed that the EFI had a close correlation with pregnancy rate in 222 patients. In 2013, Tomassetti C et al. suggested that the EFI could be used clinically to counsel infertile endometriosis patients receiving reproductive surgery in specialized centers about their post-operative conception options [9]. However, their study did not include patients receiving IVF treatment after endometriosis surgery. We believed that in the course of ovarian stimulation for IVF treatment, it would be better to predict fertility after endometriosis surgery by comparing the dosage of medication, number of oocytes retrieved, embryo quality, implantation rate, and pregnancy rate in different groups of EFI scores. Unlike the r-AFS classification, the EFI objectively evaluates factors closely 
Table 3 The embryos quality and outcome of IVF-ET

\begin{tabular}{|c|c|c|c|c|c|c|c|c|c|}
\hline \multirow[t]{2}{*}{ Characteristic } & \multirow[b]{2}{*}{ Total } & \multicolumn{4}{|c|}{ EFI } & \multicolumn{4}{|c|}{ r-AFS } \\
\hline & & $\leq 5$ score & $\geq 6$ score & $z\left(x^{2}\right)$ & $P^{c}$ & I-II stage & III-IV stage & $z\left(x^{2}\right)$ & $P^{d}$ \\
\hline No. of cycles & 199 & 84 & 115 & & & 61 & 138 & & \\
\hline No. of oocytes retrieved ${ }^{a}$ & $8.0(4.0-12.0)$ & $6.0(3.0-10.8)$ & $9.0(5.0-14.0)$ & -3.071 & 0.002 & $9.0(5.0-15.0)$ & $7.0(3.0-11.3)$ & -2.454 & 0.0 \\
\hline No. of fertilization & $5.0(3.0-9.0)$ & $4.0(2.0-8.0)$ & $6.0(4.0-10.0)$ & -3.292 & 0.001 & $6.0(4.0-10.0)$ & $5.0(2.8-8.0)$ & -2.493 & 0.013 \\
\hline No. of fertilization 2PN ${ }^{\mathrm{a}}$ & $4.0(2.0-7.0)$ & $3.5(1.0-7.0)$ & $5.0(3.0-8.0)$ & -2.735 & 0.006 & $5.0(3.0-9.0)$ & $4.0(2.0-7.0)$ & -1.851 & 0.06 \\
\hline Rate of 2PN fertilization ${ }^{\mathrm{b}}$ & 1082/1834(59.0\%) & $414 / 676(61.2)$ & $668 / 1158(57.7)$ & 2.232 & 0.135 & $379 / 671(56.5)$ & $703 / 1163(60.4)$ & 2.764 & 0.096 \\
\hline Rate of polypronucleus zygote(\%) & 154/1834(8.4\%) & $51 / 676(7.5)$ & 103/1158(8.9) & 1.012 & 0.314 & 70/671(10.4) & $84 / 1163(7.2)$ & 5.698 & 0.01 \\
\hline No. of cleavage from 2PN & $4.0(2.0-7.0)$ & $3.0(1.0-6.0)$ & $5.0(3.0-7.0)$ & -2.980 & 0.003 & $5.0(3.0-9.0)$ & $4.0(2.0-6.0)$ & -2.082 & 0.03 \\
\hline Cleavage rate from 2PN zygote ${ }^{b}$ & 1045/1236(84.5) & 391/465(84.1) & $654 / 771(84.8)$ & 0.121 & 0.728 & 372/449(82.9) & $673 / 787(85.5)$ & 0.696 & 0.404 \\
\hline Availability of embryos(\%) & $924 / 1306(70.8)$ & $347 / 496(70.0)$ & $577 / 810(71.2)$ & 0.242 & 0.623 & $310 / 475(65.3)$ & 614/831(73.9) & 10.862 & 0.00 \\
\hline Top quality embryos(\%) & 139/1045(13.3) & 53/391(13.6) & $86 / 654(13.1)$ & 0.035 & 0.852 & $52 / 372(13.9)$ & $87 / 673(12.9)$ & 0.230 & 0.632 \\
\hline No. Of embryos transferred & $2.0(2.0-2.0)$ & $2.0(1.0-2.75)$ & $2.0(2.0-2.0)$ & -1.920 & 0.055 & $2.0(2.0-3.0)$ & $2.0(2.0-2.0)$ & -2.349 & 0.019 \\
\hline Implantation rate $(\%)^{\mathrm{b}}$ & 120/386(31.1) & $37 / 148(25.0)$ & 83/238(34.9) & 4.153 & 0.042 & 43/131(32.8) & $77 / 255(30.2)$ & 0.279 & 0.597 \\
\hline Clinical pregnancy rate(\%) & $85 / 199(42.7)$ & 24/84(28.6) & 61/115(53.0) & 11.881 & 0.001 & $31 / 61(50.8)$ & $54 / 138(39.1)$ & 2.362 & 0.124 \\
\hline Accumulation rate of pregnancy ${ }^{b}$ & 107/199(53.8) & 33/84(39.2) & 74/115(64.3) & 12.266 & 0.000 & $37 / 61(60.7)$ & 70/138(50.7) & 1.678 & 0.195 \\
\hline
\end{tabular}

Note: Rate of 2PN fertilisation = number of $2 \mathrm{PN}$ zygotes/number of oocytes retrieved; rate of polypronucleus zygotes = number of polypronucleus zygotes/number of oocytes retrieved; rate of cleavage from $2 \mathrm{PN}=$ number of cleavages from $2 \mathrm{PN} /$ number of zygotes; embryo availability rate $=$ (number of frozen embryos + number of ET embryos)/number of cleavage-stage embryos; top-quality embryos = number of top-quality embryos/number of cleavage-stage

embryos from 2PN; implantation rate = number of gestational sacs/number of ET embryos; clinical pregnancy rate = number of pregnancy cycles/number of embryo transfer cycles; accumulation rate of pregnancy = number of pregnancy cycles for fresh transferred embryos or freeze-thawed transferred embryos in a single cycle of ovulation stimulation/number of cycles for ovulation stimulation.

Definition of top-quality embryo: seven to eight blastomeres of equal size and less than $10 \%$ fragmentation can be seen on day 3 ( Alpha Scientists in Reproductive Medicine and ESHRE Special Interest Group of Embryology, 2011) [19].

${ }^{a}$ The non-normally distributed data are presented as median (25th-75th percentile). The non-parametric Mann-Whitney test was performed to calculate the Z-score.

${ }^{\mathrm{b}}$ The chi-squared test was performed to compare rates between groups.

${ }^{c}$ Comparison between the EFI score $\leq 5$ and $\geq 6$ groups. (score $\leq 5$ vs score $\geq 6$ ).

${ }^{\mathrm{d}}$ Comparison between the r-AFS stage I-II and stage III-IV groups (stage I-II vs stage III-IV).

associated with female fertility, such as fallopian tube, tubal fimbria, and ovarian function. It incorporates the LF score which can evaluate the reproductive potential of pelvic organs and comprehensively includes several objective factors, such as patient's age, duration of infertility, and pregnancy history. According to our data, the AUC ROC for embryo transfer during a fresh IVF cycle was only 0.641 using the EFI score. Patients with a score of 6 accounted for the largest proportion in our study. Patients with a score $\geq 6$ had a significantly higher pregnancy rate than patients with a score $\leq 5$. This is slightly different from the study of Adamson et al. According to their study, patients with a score of 7 accounted for the largest proportion [8]. Patients with scores of 8-10 had higher cumulative pregnancy rates than patients with scores of 5-7. A plausible explanation for this difference is that the participants in the two studies used different methods to conceive. The participants in our study were patients receiving IVF treatment after surgery. Therefore, the fallopian tube factors had little impact on the outcome of their IVF treatment, which could have lowered the cut-off point in the EFI in comparison to the patients who planned pregnancy naturally after the surgery.
The EFI incorporates patient age and duration of infertility. As shown in Table 2, no significant differences were observed in the baseline BMI and basal sex hormone levels between patients with EFI scores $\leq 5$ and $\geq 6$. The antral follicle count and the starting dose of $\mathrm{Gn}$ were different between the two groups (total Gn dose was also slightly different). This suggests that physicians estimated the starting Gn dose based on BMI, basal sex hormone levels, and antral follicle count in patients with different EFI scores. Therefore, both starting and total Gn doses were affected by oocyte reserve. The number of oocytes retrieved was also relatively higher in the $\geq 6$ score group. However, although the rates of available embryos and top-quality embryos showed no significant differences between groups, the implantation rate and pregnancy rate were higher in the $\geq 6$ score group, suggesting that a score of 6 can be considered an appropriate cut-off point when assessing EFI in endometriosis patients. The pregnancy rate was also higher in patients with score $\geq 6$.

In summary, the EFI incorporates age and duration of infertility; hence, it objectively evaluates the function of reproductive organs better than $\mathrm{r}$-ASF. It has a relatively good predictive power for pregnancy outcomes for 
patients receiving IVF-ET treatment after laparoscopic surgery. The implantation rate and pregnancy rate were higher in patients with EFI score $\geq 6$ than those with EFI score $\leq 5$. These data provide an important reference to predict the post-operative pregnancy outcome for endometriosis patients, which is the most valuable conclusion of this study.

This study has several limitations. First, there were no significant differences in the rate of available embryos or the rate of top-quality embryos between EFI groups or between r-ASF groups. However, the implantation rate and pregnancy rate were significantly different between groups, suggesting that endometriosis can not only affect the ovaries and quality of oocytes but also the uterine endometrium for embryo implantation. A more convincing support of the implantation environment theory would be if we followed up the development of blastocysts by evaluating day 5 and day 6 blastocysts after implantation. Second, we used pregnancy/non-pregnancy as the relevant outcome to calculate the sample size without considering fertilisation or available embryos. Therefore, as shown in Table 3, the reason the r-AFS stage III-IV group had a low rate of polypronucleus zygotes and a high number of available embryos were that the r-AFS stage I-II group was relatively small. Third, this study failed to demonstrate whether starting an IVF cycle earlier after laparoscopic surgery can improve the specificity and sensitivity of the EFI. Fourth, severe uterine abnormalities, such as uterine fibroids, adversely affect pregnancy. However, the EFI does not incorporate such uterine conditions, a limitation of the scoring system. We acknowledge the impact of uterine conditions on pregnancy and the fact that EFI does not incorporate them. Therefore, to avoid any bias, we only included cases in this study that did not present with significant uterus fibroids as evaluated by laparoscopy and ultrasound. Finally, this was a retrospective study with the calculated cut-off EFI score of 6 . Although the included sample size achieved the requirement for diagnostic tests, it will be necessary to conduct randomised, prospective studies with large numbers of patients to validate and evaluate the EFI and its cut-off point. It is better to analyse pregnancy probability in each stage of the EFI with a larger sample size.

\section{Conclusions}

It suggests that the EFI has more predictive power for IVF outcomes in endometriosis patients than the r-AFS classification. The clinical pregnancy rate was higher in patients with EFI $\geq 6$ score than with EFI $\leq 5$ score.

\section{Competing interests}

The authors declare that they have no competing interests.

\section{Authors' contributions}

WJW conceived the idea and design of the project, collected all cases, did the statistical analysis, drafted the manuscript, edited the final document and acquired funding. RQL collected cases. TFF provided access to the statistical analysis and patient data. LLH and NYO collected cases. LAW collected cases and performed laparoscopic surgery. QXZ provided access to the statistical analysis. DZY acquired funding. All authors read and approved the final manuscript.

\section{Acknowledgments}

This study was supported by the Science Research of Population and Family Planning Commission of Guangdong Province in China (Grant No. 2009231), the Science Technology Research of development Project of Guangdong Province (Grant No. 2010B031600043) and the Science Technology Research Project of Guangzhou city (2010Y1-C431). The authors wish to express their gratitude to G. David Adamson, MD for editing and giving them permission to use the updated version of the EFI, and to Meiqing Xie, MD, PhD, and Shunjia Hong, MD, PhD, for their laparoscopic surgery, and to Huang Baoyun for data collection, and to John P. , and Suja T. for English-language edit. The manuscript was also edited by AJE (American Journal Experts).

\section{Author details}

'Department of Obstetrics \& Gynecology, Reproductive medicine centre, Sun Yat-Sen Memorial Hospital, Sun Yat-Sen University, No.107 Yanjiang Xi Road, Guangzhou 510120, P. R. China. ${ }^{2}$ New Hope Fertility Center, Av.De Praia Grande No.409, China Law Building, 3 Andar BC, Macau, P. R. China. ${ }^{3}$ Endocrinology division, Department of Obstetrics \& Gynecology, Sun Yat-Sen Memorial Hospital, Sun Yat-Sen University, No.107 Yanjiang Xi Road, Guangzhou 510120, P. R. China.

Received: 31 August 2013 Accepted: 7 December 2013

Published: 11 December 2013

\section{References}

1. Giudice LC, Kao LC: Endometriosis. Lancet 2004, 364:1789-1799.

2. Hummelshoj L, Prentice A, Groothuis P: Update on endometriosis. Womens Health (Lond Engl) 2006, 2:53-56.

3. Counseller VS, Crenshaw Jr JL: A clinical and surgical review of endometriosis. Am J Obstet Gynecol 1951, 62:930-942.

4. The American Fertility Society: Revised American Fertility Society classification of endometriosis: 1985. Fertil Steril 1985, 43:351-352.

5. Canis M, Donnez JG, Guzick DS, Halme JK, Rock JA, Schenken RS, Vemon MW: Revised American Society for Reproductive Medicine classification of endometriosis: 1996. Fertil Steril 1997, 67:817-821.

6. Adamson GD: Endometriosis classification: an update. Curr Opin Obstet Gynecol 2011, 23:213-220.

7. Vercellini P, Fedele L, Aimi G, De Giorgi O, Consonni D, Crosignani PG: Reproductive performance, pain recurrence and disease relapse after conservative surgical treatment for endometriosis: the predictive value of the current classification system. Hum Reprod 2006, 21:2679-2685.

8. Adamson GD, Pasta DJ: Endometriosis fertility index: the new, validated endometriosis staging system. Fertil Steril 2010, 94:1609-1615.

9. Tomassetti C, Geysenbergh B, Meuleman C, Timmerman D, Fieuws S, D'Hooghe T: External validation of the endometriosis fertility index (EFI) staging system for predicting non-ART pregnancy after endometriosis surgery. Hum Reprod 2013, 28:1280-1288.

10. Organization WHO: WHO laboratory manual for the Examination and processing of human semen. Geneva, Switzerland: WHO Press; 2010:224.

11. Tavmergen E, Ulukus M, Goker EN: Long-term use of gonadotropinreleasing hormone analogues before IVF in women with endometriosis. Curr Opin Obstet Gynecol 2007, 19:284-288.

12. Somigliana E, Vercellini P, Vigano P, Ragni G, Crosignani PG: Should endometriomas be treated before IVF-ICSI cycles. Hum Reprod Update 2006, 12:57-64.

13. Romao GS, Araujo MC, de Melo AS, de Albuquerque Salles Navarro PA, Ferriani RA, Dos RRM: Oocyte diameter as a predictor of fertilization and embryo quality in assisted reproduction cycles. Fertil Steril 2010, 93:621-625.

14. Scott L, Alvero R, Leondires M, Miller B: The morphology of human pronuclear embryos is positively related to blastocyst development and implantation. Hum Reprod 2000, 15:2394-2403. 
15. Chen H, Wang W, Mo Y, Ma Y, Ouyang N, Li R, Mai M, He Y, BodombossouDjobo MM, Yang D: Women with high telomerase activity in luteinised granulosa cells have a higher pregnancy rate during in vitro fertilisation treatment. J Assist Reprod Genet 2011, 28:797-807.

16. Donner A: Approaches to sample size estimation in the design of clinical trials-a review. Stat Med 1984, 3:199-214.

17. Lerman J: Study design in clinical research: sample size estimation and power analysis. Can J Anaesth 1996, 43:184-191.

18. Bossuyt PM, Reitsma JB, Bruns DE, Gatsonis CA, Glasziou PP, Irwig LM, Moher D, Rennie D, de Vet HC, Lijmer JG: The STARD statement for reporting studies of diagnostic accuracy: explanation and elaboration. Ann Intern Med 2003, 138:W1-12.

19. Balaban B, Brison D, Calderón G, Catt J, Conaghan J, Cowan L, Ebner T, Gardner D, Hardarson T, Lundin K, Cristina Magli M, Mortimer D, Mortimer S, Munné S, Royere D, Scott L, Smitz J, Thornhill A, van Blerkom J, Van den Abbeel E: The Istanbul consensus workshop on embryo assessment: proceedings of an expert meeting. Hum Reprod 2011, 26:1270-1283.

20. Opoien HK, Fedorcsak P, Omland AK, Abyholm T, Bjercke S, Ertzeid G, Oldereid N, Mellembakken JR, Tanbo T: In vitro fertilization is a successful treatment in endometriosis-associated infertility. Fertil Steril 2012, 97:912-918.

21. Fujishita AKKN, Masuzaki HIT: Influence of pelvic endometriosis and ovarian endometrioma on fertility. Gynecol Obstet Invest 2002, 53:40-45.

doi:10.1186/1477-7827-11-112

Cite this article as: Wang et al: Endometriosis fertility index score maybe more accurate for predicting the outcomes of in vitro fertilisation than r-AFS classification in women with endometriosis. Reproductive Biology and Endocrinology 2013 11:112.

\section{Submit your next manuscript to BioMed Central and take full advantage of:}

- Convenient online submission

- Thorough peer review

- No space constraints or color figure charges

- Immediate publication on acceptance

- Inclusion in PubMed, CAS, Scopus and Google Scholar

- Research which is freely available for redistribution

Submit your manuscript at www.biomedcentral.com/submit
() Biomed Central 